\title{
Correction to: Short-term dietary restriction in old mice rejuvenates the aging-induced structural imbalance of gut microbiota
}

\author{
Ting Zeng $\cdot$ Hui Cui $\cdot$ Duozhuang Tang $\cdot$ George B. Garside $\cdot$ Yiting Wang \\ Jianying Wu $\cdot$ Zhendong Tao $\cdot$ Liu Zhang $\cdot$ Si Tao
}

Published online: 9 September 2019

(C) The Author(s) 2019

\section{Correction to: Biogerontology https://doi.org/10.1007/s10522-019-09830-5}

In the original publication of the article, the alphabets in figures were published in upper case and mismatched with the figure legends.
The original article can be found online at https:// doi.org/10.1007/s10522-019-09830-5.

T. Zeng $\cdot$ H. Cui $\cdot$ J. Wu

Jiangxi Key Laboratory of Clinical and Translational Cancer Research, Department of Oncology, The Second Affiliated Hospital of Nanchang University, Jiangxi,

China

T. Zeng $\cdot$ H. Cui $\cdot$ J. Wu $\cdot$ S. Tao $(\bowtie)$

Department of Oncology, The Second Affiliated Hospital of Nanchang University, Min-De Road. 1,

330006 Nanchang City, Jiangxi Province, China

e-mail: ndefy11188@ncu.edu.cn

D. Tang $\cdot$ Y. Wang

Department of Hematology, The Second Affiliated

Hospital of Nanchang University, Jiangxi, China
The corrected Figs. 1, 2, 3, 4 are given in this Correction.

G. B. Garside

Leibniz Institute on Aging - Fritz Lipmann Institute (FLI),

Jena, Germany

Z. Tao

Department of Medical Laboratory Medicine, Jiangxi

Province Hospital of Integrated Chinese \& Western

Medicine, Jiangxi, China

L. Zhang

Intensive Care Unit, Peking University People's Hospital, Beijing, China 

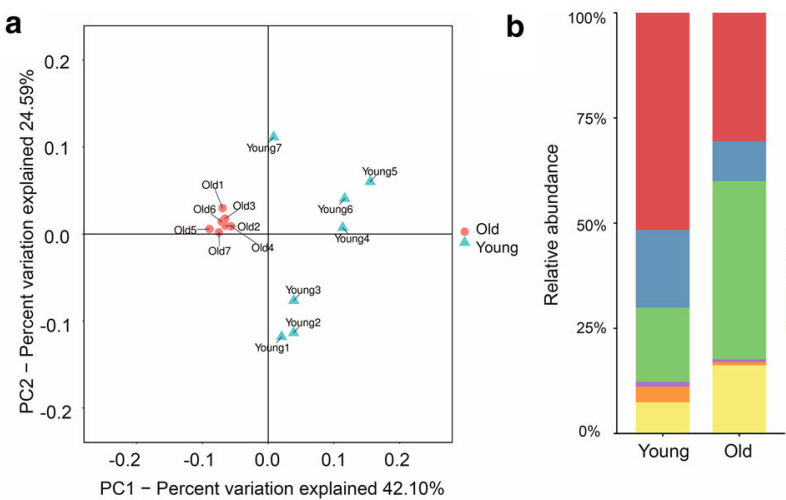

Taxonomy

- k_Bacteria; $p$ _Bacteroidetes

k_Bacteria;p_Epsilonbacterae

k_Bacteria; P Proteobacteria

k_Bacteria; ;__Verrucomicrobi

Others

c

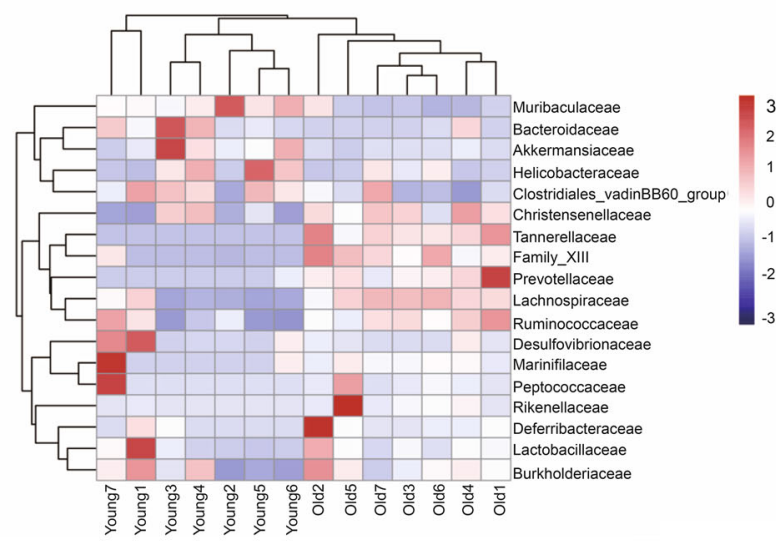

d

$$
\begin{aligned}
& \text { Young } \\
& \text { Old }
\end{aligned}
$$

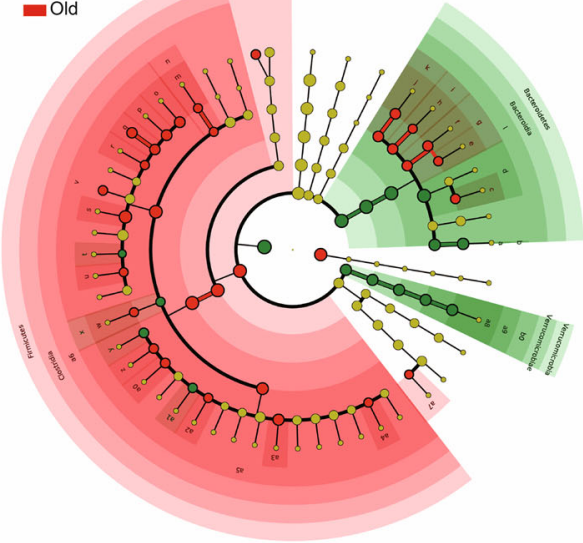

e

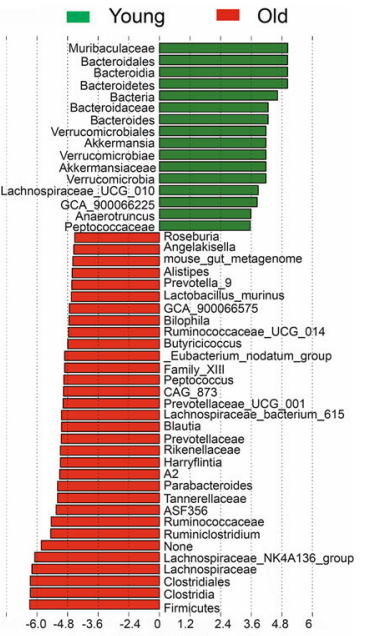

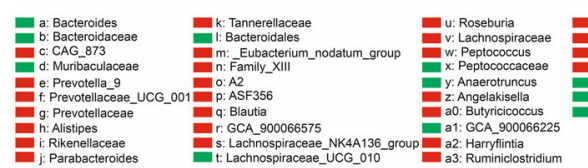

a4: Ruminococcaccae__UCG_o14 LDA SCORE (log 10)

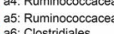

Bab: Clostriciales
ar: Bilophila

as: Akkermansia
ag: Akermansiace

ag: Akkermansiaceoea
bo: Verrucomicrobiales

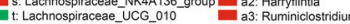


4Fig. 1 Alterations in the fecal microbial community structure of aging mice. Fecal samples of 2 months old (young) and 20-22 months old (old) mice were collected for analysis ( $\mathrm{n}=7$ samples per group). a $\beta$-diversity analysis. The results of unweighted UniFrac PCoA were shown. b Relative abundance of bacteria at phylum level. The ratio of the average OTU for each group was shown. c Heatmap based on the relative abundance at family level. d Taxonomic cladogram from LEfSe showing differences in fecal taxa. Dot size is proportional to the abundance of the taxon. Letters correspond to the following taxa: a: Bacteroides, b: Bacteroidaceae, c: CAG_873, d: Muribaculaceae, e: Prevotella_9, f: Prevotellaceae_UCG_001, g: Prevotellaceae, h: Alistipes, i: Rikenellaceae, j: Parabacteroides, k: Tannerellaceae, 1: Bacteroidales, m: Eubacterium_nodatum_group, n: Family_XIII, o: A2, p: ASF356, q:Blautia, r: GCA_900066575, s: Lachnospiraceae_NK4A136_group, t: Lachnospiraceae_UCG_010, u: Roseburia, v: Lachnospiraceae, w: Peptococcus, x: Peptococcaceae, y: Anaerotruncus, z: Angelakisella, a0: Butyricicoccus, a1: GCA_900066225, a2: Harryflintia, a3: Ruminiclostridium, a4: Ruminococcaceae_UCG_014, a5: Ruminococcaceae, a6: Clostridiales, a7: Bilophila, a8: Akkermansia, a9: Akkermansiaceae, b0: Verrucomicrobiales. e LDA scores computed for differentially-abundant taxa in the fecal microbiomes of young and old mice. Length indicates effect size associated with a taxon. $\mathrm{p}=0.05$ for the Kruskal-Wallis test; LDA score $>2$ 

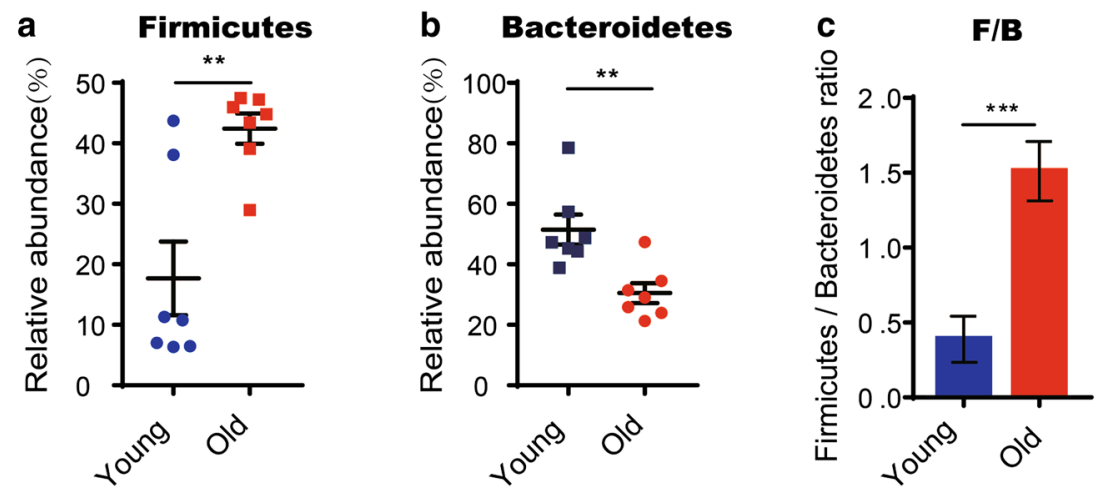

d Christensenellaceae
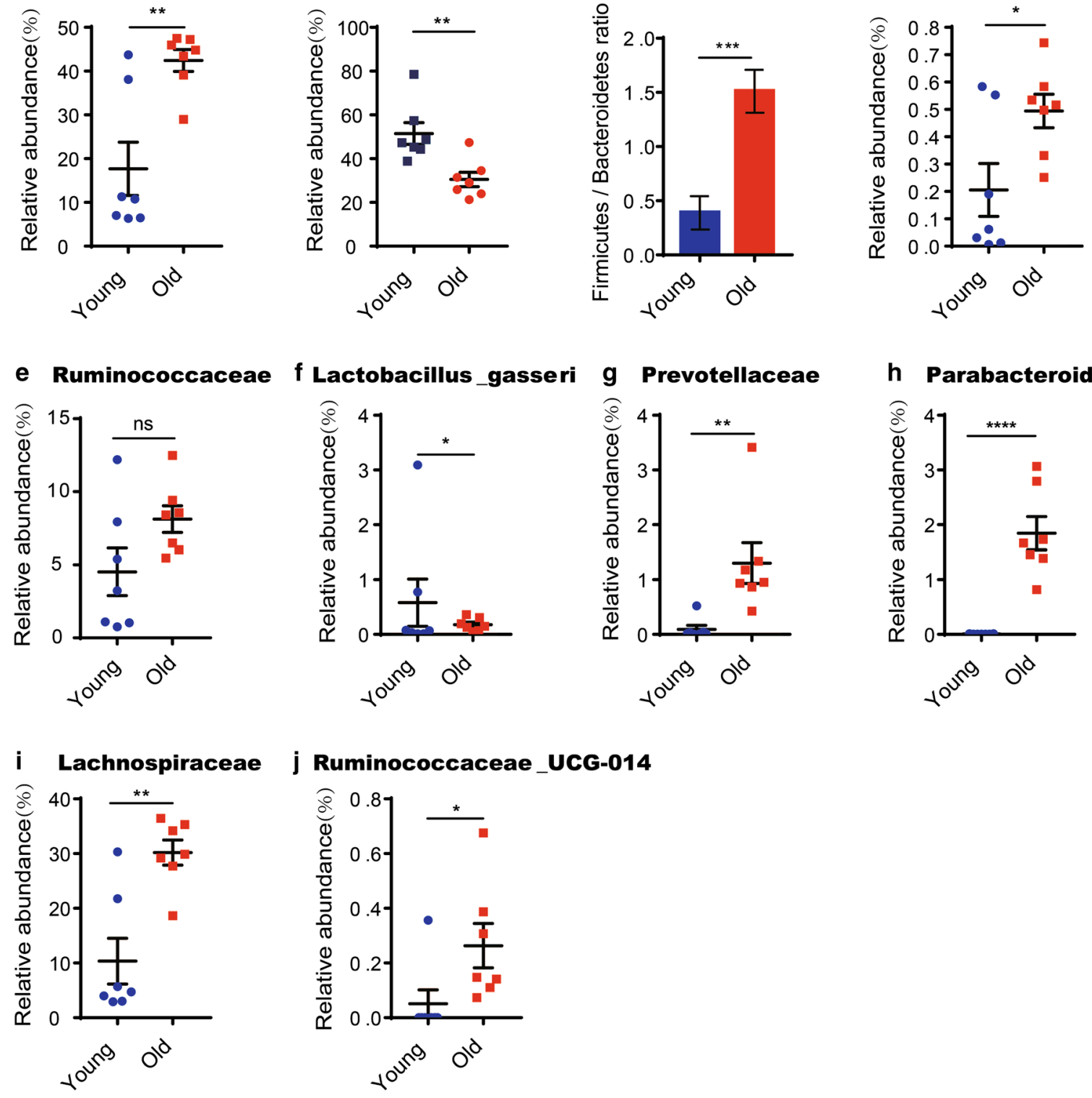

Fig. 2 Lipid-promoting and pro-inflammatory bacteria are enriched in aging mice. Fecal samples of 2 months old (young) and 20-22 months old (old) mice were collected for analysis ( $\mathrm{n}=7$ samples per group). a, b, d-j Relative abundance based on OTUs of intestinal bacteria taxa which are significantly changed in old mice. Note that these taxa were all lipid- promoting or pro-inflammatory bacteria. c Ratio of Firmicutes/ Bacteroidetes based on relative abundance of OTUs. Note a significant increase in the old mice compared to the young ones. Results were displayed as mean \pm SEM. $* p<0.05$; $* * \mathrm{p}<0.01 ; * * * \mathrm{p}<0.001 ; * * * * \mathrm{p}<0.0001$ by unpaired twotailed Student's t test 

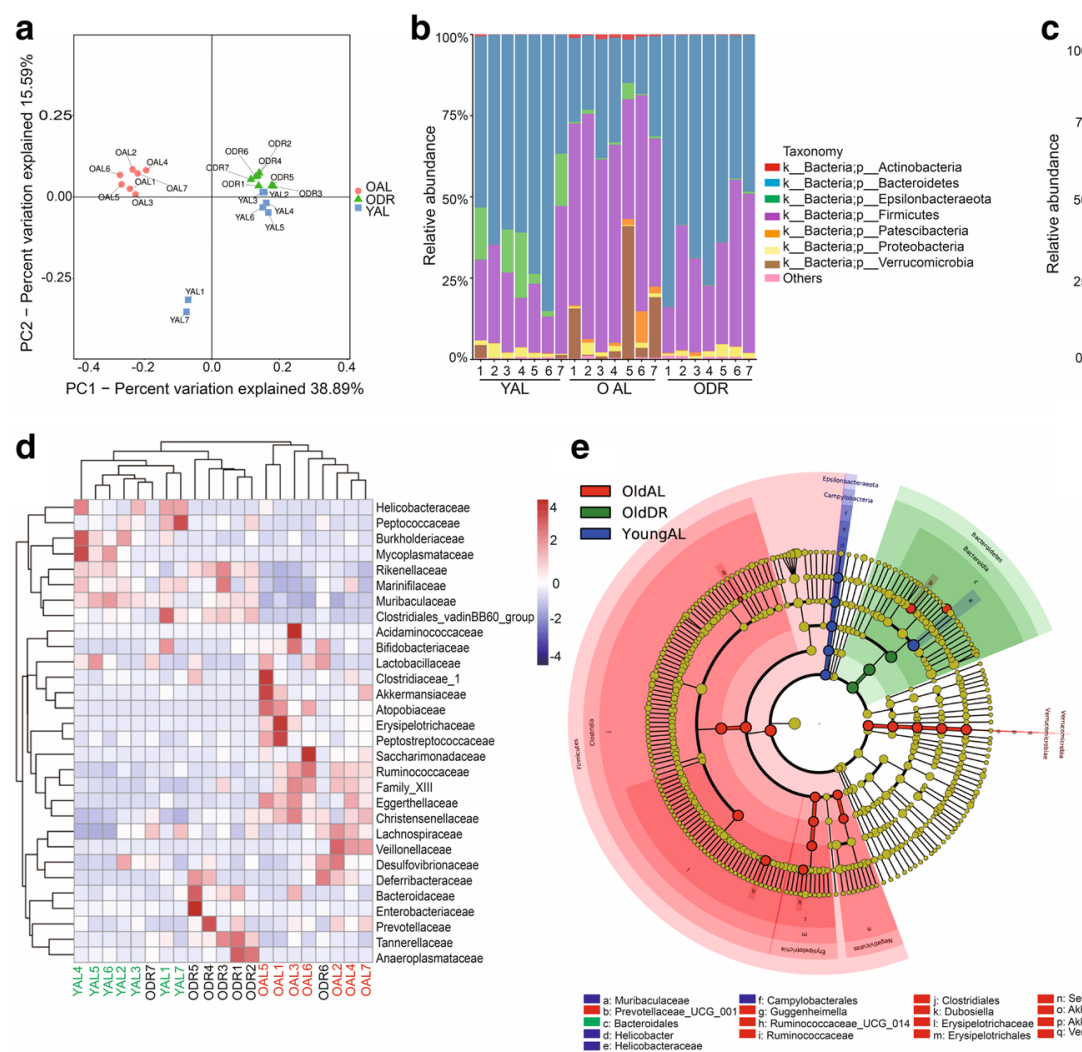

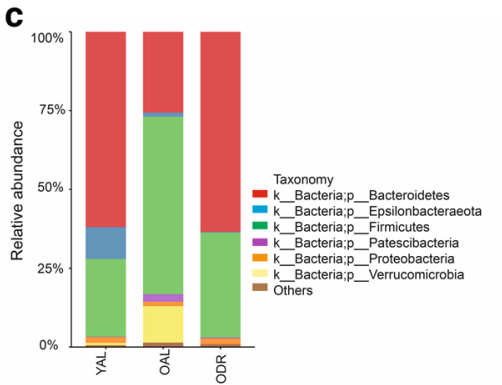

f

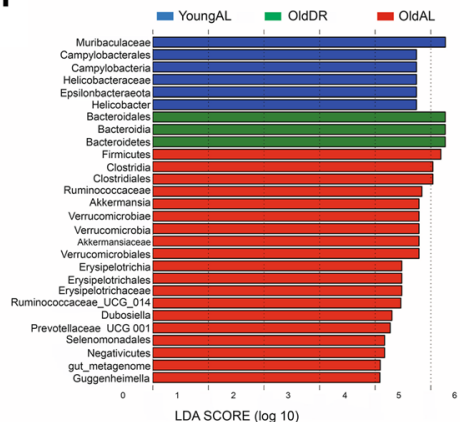

Fig. 3 Short-term DR in old mice rejuvenates aging induced structural rearrangement of gut microbiota. 20-22 months old mice were treated with DR or AL diet for 2 months. Fecal samples of the following groups were collected for analysis: YAL (2 months old mice fed with ad libitum), OAL (22-24 months old mice fed with ad libitum), and ODR (22-24 months old mice pre-treated with DR for 2 months before sample collection) ( $n=7$ samples per group). a $\beta$ diversity analysis. The results of unweighted UniFrac PCoA of indicated groups were shown. b Relative abundance of bacteria at phylum level of individual sample based on OTUs. $\mathbf{c}$ The ratio of relative abundance at phylum level based on the average OTUs in each group. d Heatmap showing clustering of each sample at family level based on the relative abundance of OTUs. Note that hierarchical clustering shown that samples of ODR and YAL tend to cluster together. e Taxonomic cladogram from LEfSe showing differences in fecal taxa. Dot size is proportional to the abundance of the taxon. Letters correspond to the following taxa: a: Muribaculaceae, b: Prevotellaceae_UCG_001, c: Bacteroidales, d: Helicobacter, e: Helicobacteraceae, f: Campylobacterales, g: Guggenheimella, h: Ruminococcaceae_UCG_014, i: Ruminococcaceae, j: Clostridiales, k: Dubosiella, 1: Erysipelotrichaceae, m: Erysipelotrichales, n: Selenomonadales, o: Akkermansia, p: Akkermansiaceae, q: Verrucomicrobiales. f LDA scores computed for differentially-abundant taxa in the fecal microbiomes of young (blue) old DR (green) and old AL (red). Length indicates effect size associated with a taxon. $p=0.05$ for the Kruskal-Wallis test; LDA score $>2$ 

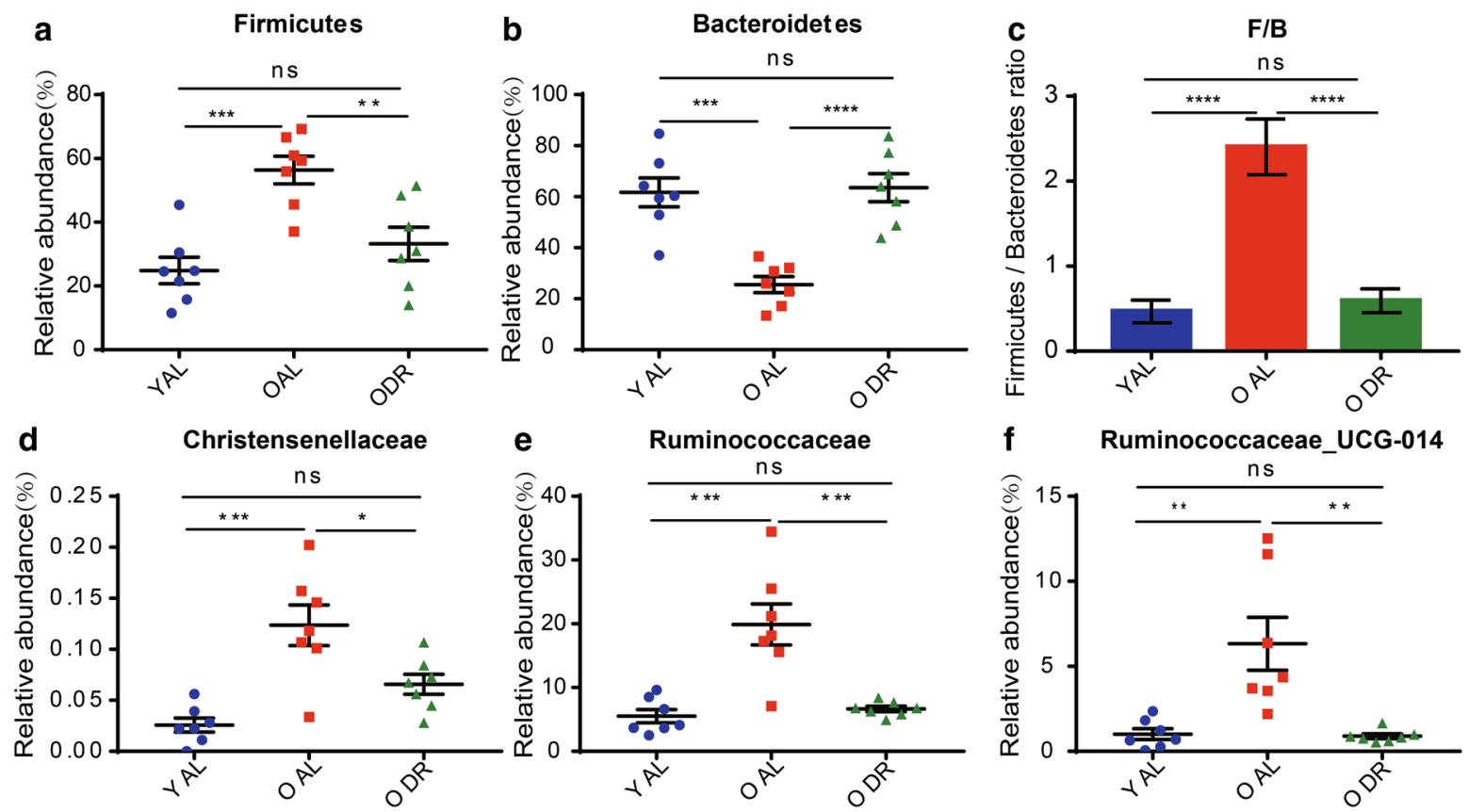

\section{g Clostridiales_vadin BB60_group $\mathbf{h}$}

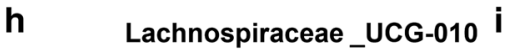

i [Eubacterium]_coprostanoligenes_group
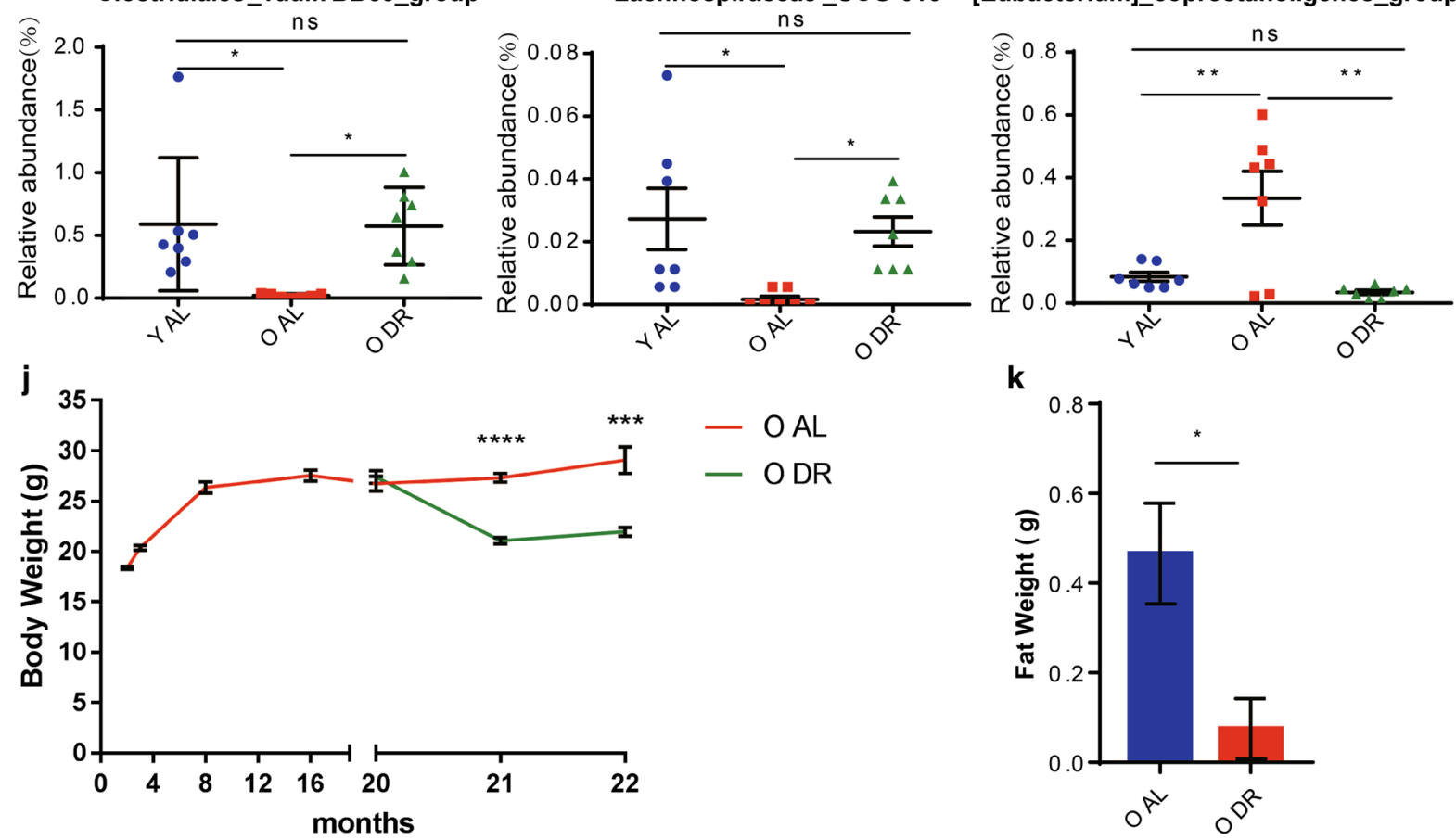

Fig. 4 Short-term DR reverted compositional alterations of bacterial taxa associated with obesity and inflammation in aging mice. 20-22 months old mice were treated with DR or AL diet for 2 months. Fecal samples of the following groups were collected for analysis: YAL (2 months old mice fed with ad libitum), OAL (22-24 months old mice fed with ad libitum), and ODR (22-24 months old mice pre-treated with DR for 2 months before sample collection) ( $\mathrm{n}=7$ samples per group). a, b, d-i Relative abundance based on OTUs of intestinal bacteria taxa. c Ratio of Firmicutes/Bacteroidetes based on relative abundance of OTUs. Note that DR significantly rejuvenated all alterations of indicated taxa in aging mice. $\mathbf{j}$, k Body and belly fat weight of indicated groups. Note a significant reduction upon DR. Results were displayed as mean \pm SEM. $\quad * \mathrm{p}<0.05 ; \quad * * \mathrm{p}<0.01 ; \quad * * * \mathrm{p}<0.001$; $* * * * \mathrm{p}<0.0001$ by unpaired two-tailed Student's t test 
Open Access This article is distributed under the terms of the Creative Commons Attribution 4.0 International License (http://creativecommons.org/licenses/by/4.0/), which permits unrestricted use, distribution, and reproduction in any medium, provided you give appropriate credit to the original author(s) and the source, provide a link to the Creative Commons license, and indicate if changes were made.

Publisher's Note Springer Nature remains neutral with regard to jurisdictional claims in published maps and institutional affiliations. 